\title{
AUX ORIGINES DU MUSÉE D'ENTREPRISE : MUSÉES INDUSTRIELS ET COMMERCIAUX
}

\author{
François Mairesse ${ }^{1}$
}

Je souhaiterais établir, dans cet article, à travers la création de quelques musées emblématiques comme le Conservatoire national des Arts et Métiers ou le Victoria \& Albert Museum et de deux catégories spécifiques d'institutions - les musées d'art industriels et les musées commerciaux -, le fait que les musées d'entreprise, loin d'apparaitre comme une excroissance un peu embarrassante dans le monde des musées, s'inscrivent au cœur de son histoire et de ses enjeux actuels.

On ne peut pas dire que la catégorie des «musées d'entreprise» soit particulièrement valorisée au sein du monde des musées. On place plutôt ces derniers à la fin des typologies muséales, dans la catégorie « autres musées » (Labourdette, 2015), en marge des établissements publics les plus prestigieux comme le Louvre ou le Muséum national d'histoire naturelle. Une catégorie un peu honteuse : le musée est par définition une organisation sans but lucratif (ICOM, 2016) et le monde muséal s'est souvent méfié des entreprises commerciales ; il n'accepte ces dernières qu'avec suspicion. Ainsi, une société comme le musée Grévin n'est, aux yeux du Conseil international des musées (ICOM), pas

1 François Mairesse est professeur au département de Médiation culturelle, Université Sorbonne nouvelle-Paris 3 et chercheur au Centre de recherche sur le lien social (CERLIS) et au Laboratoire d'excellence Industries culturelles et création artistique (ICCA).

Recherches en communication, $\mathrm{n}^{\circ} 45$ - Article publié le 06/03/2018 
reconnue comme un musée. La définition que 1'ICOM donne du musée semble également assez mal adaptée au contexte des musées d'entreprise. Le musée est ainsi présenté comme : « une institution permanente sans but lucratif, au service de la société et de son développement, ouverte au public, qui acquiert, conserve, étudie, expose et transmet le patrimoine matériel et immatériel de l'humanité et de son environnement à des fins drétudes, d)éducation et de délectation » (ICOM, 2016). On peut accepter que les musées d'entreprise soient sans but lucratif et remplissent les mêmes fonctions techniques que celles des musées (acquisition, conservation, exposition - on peut s'interroger sur l'étude) mais il est plus difficile d'y voir des établissements conçus à des fins d'études et d'éducation ou de délectation. Ces collections de machines-outils, de voitures ou de bonbons, souvent conçues et présentées comme des faire-valoir de l'entreprise qui les expose, quelquefois rassemblées par un patron ou un ingénieur collectionneur, parfois constituées dans le cadre d'une politique de marque, apparaissent ainsi souvent éloignées des objectifs de connaissance ou de préservation du patrimoine que l'on associe plus étroitement au monde des musées depuis leur origine. Il est évidemment difficile, dans un tel contexte, d'associer les musées d'entreprise à la quête humaniste que l'on trouve dès le $\mathrm{XVI}^{\mathrm{e}}$ siècle au sein des galeries ou des cabinets de curiosités.

L'histoire des musées est cependant plus complexe : si les fonctions de cette institution participent d'emblée à une logique de recherche, de préservation ou d'éducation, on les associe aussi, depuis au moins le XVIII ${ }^{\mathrm{e}}$ siècle, à des enjeux économiques. L'origine des musées d'entreprise, à cet égard, peut être liée à d'autres établissements importants dont l'évolution, tout au long des deux derniers siècles, montre l'importance du développement économique dans les conceptions muséales. Je souhaiterais établir, dans cet article, à travers la création de quelques musées emblématiques comme le Conservatoire national des Arts et Métiers ou le Victoria \& Albert Museum et de deux catégories spécifiques d'institutions - les musées d'art industriels et les musées commerciaux -, le fait que les musées d'entreprise, loin d'apparaître comme une excroissance un peu embarrassante dans 
le monde des musées, s'inscrivent au cœur de son histoire et de ses enjeux actuels. Il convient certes de distinguer les musées d'entreprise actuels de ces premiers musées techniques ou d'arts appliqués dont la logique diffère sensiblement. Il n'empêche que - et c'est le propos de cet article - certains des projets au cœur des musées d'entreprise, loin de constituer une particularité, dérivent des projets portés par ces établissements pionniers décrits dans cet article. Curieux paradoxe que celui de ces établissements d'initiative essentiellement publique, partageant avec les musées d'entreprise actuels - créés par le secteur privé - des objectifs partiellement similaires. Cet article envisage le développement du musée à partir de son projet (Mairesse, 2002), et non à partir de l'histoire des collections. Il ne saurait être question de nier l'initiative de certains patrons collectionneurs, cédant le fruit de leur passion, parfois liée à leur activité entrepreneuriale, à leur entreprise. Une telle pratique ne diffère guère de celle que l'on peut retrouver dans de nombreux autres domaines plus classiques, qu'il s'agisse de beaux-arts ou de techniques. L'originalité de la forme du musée d'entreprise, en revanche, tient moins à la personnalité de son patron ou à la qualité de sa collection qu'à des enjeux partiellement différents liés à l'activité du musée et que l'on pourrait caractériser comme essentiellement pratique et économique. C'est aspect similaire de ce type de projets que l'on retrouve aussi à l'origine des premiers musées industriels.

\section{Lumières, économie et premiers musées industriels}

On sait l'importance des collections dans le projet de développement de la science moderne, notamment dans la pensée de Bacon (Pousseur, 1988), et c'est dans cette optique que sont créés, dès le XVII ${ }^{\mathrm{e}}$ siècle, nombre de cabinets, notamment celui de la Royal Society de Londres (Bredekamp, 1997, Rasse, 2017). C'est une logique relativement similaire qui conduit Leibniz à recommander la création de Kunst- und Raritätenkammern pour l'établissement de sociétés scientifiques. Mais dès cette époque, les arguments apportés par le savant allemand, s'ils visent le développement des connaissances, sont aussi de nature 
mercantile, comme il l'écrit en 1680 à l'électeur de Hanovre : « une seule invention, soit mécanique, soit chimique, qui ailleurs ne vaudra rien, appliquée là rapportera peut-être dix ou douze mille thalers de rentes »(Foucher de Careil, t.7, p. 143). Le savant allemand sait pertinemment que la connaissance, seule, ne convaincra pas toujours ses protecteurs sans l'espoir de retombées pratiques susceptibles d'amortir les investissements consentis. Le raisonnement du savant, préalable à la Révolution industrielle, se positionne d'emblée sur le terrain de la richesse des nations, que tous cherchent à développer et dont l'analyse d'Adam Smith, un siècle plus tard, marque les débuts de l'économie classique. L'ouvrage majeur de ce dernier parait en 1776, soit une vingtaine d'années après la création du British Museum (1753) et dix-huit ans avant celle du Louvre (1793). Aucun de ces deux musées ne participe directement à l'économie nationale, même si des arguments économiques ont été utilisés lors du débat autour de la création du Louvre, à la Révolution (Poulot, 1997). En revanche, la création du Conservatoire national des Arts et Métiers et de son musée, en 1794, à l'initiative de l'Abbé Grégoire, vise à contribuer directement aux efforts nationaux pour enrichir le territoire. Un conservatoire pour réunir « tous les outils et machines nouvellement inventés ou perfectionnés, [qui] va éveiller la curiosité \& l'intérêt et vous verrez dans tous les genres des progrès très rapides » (Grégoire, 1794, p. 8). L'histoire de ce musée révolutionnaire débute un demi-siècle plus tôt et s'inscrit dans le sillage du développement économique lié aux grandes manufactures de l'époque. L'ingénieur mécanicien Vaucanson, spécialiste des automates et inspecteur des manufactures, a alors installé dès 1746, en l'hôtel de Mortagne et pour le compte du Roi, une collection ouverte à un public spécialisé, constituée de machines et de modèles visant à perfectionner diverses industries, notamment celle de la soie. À la mort de Vaucanson, les collections continuent d'être exploitées et trouvent naturellement leur chemin au sein de l'ambitieux projet de Conservatoire (Jacomy, 1995). Ce mouvement d'émulation industrielle se poursuit de multiples manières et va donner lieu à la création, d'une part, des musées 
d'art industriels et des premiers musées d'entreprises, d'autre part des musées commerciaux.

Chacun cherche donc à perfectionner ses productions pour développer la richesse nationale, et l'une des initiatives marquantes en la matière est l'organisation d'expositions des produits de l'industrie française. La première de ces expositions a lieu à l'initiative du Gouvernement et de son ministre de l'intérieur, Nicolas-François de Neufchâteau, en 1798. Elles vont connaître onze éditions, jusqu'en 1849 (Colmon, 1855), avant de laisser la place à l'organisation d'expositions universelles, dont la première - au Crystal Palace de Londres - remonte à 1851. Certains des catalogues de ces musées éphémères sont d'ailleurs intitulés « musées industriels » (Société d'ingénieurs et al., 1849). D'autres institutions contemporaines, comme la Société d'encouragement pour l'industrie nationale créée en 1801 (elle existe toujours), semblent également avoir initié en leurs locaux, durant quelques années, un musée industriel (Société d'encouragement, 1810). Il s'agit de présenter, dans ce contexte, les nouvelles inventions des principaux producteurs, souvent dans un cadre prestigieux, comme au Champ de Mars, au Louvre ou aux Champs Élysées, même si le public visé est prioritairement constitué par les constructeurs et les producteurs, et non par le grand public. Cette mode des expositions va donner lieu, tout au long du XIX ${ }^{\mathrm{e}}$ siècle, à l'émergence de plusieurs types d'institutions un peu partout en Europe, à commencer par la création d'autres musées industriels conçus selon les principes du Conservatoire national des Arts et Métiers, comme celui de Bruxelles, mais aussi à des musées d'art industriel et, semblet-il, aux premiers musées d'entreprise. Le Musée de l'Industrie de Bruxelles, fondé en 1827 sous le gouvernement hollandais, a ainsi pour modèle l'établissement parisien (Michel, 1967). Cette institution connaît des débuts difficiles et doit son essor à la personnalité de son second directeur, Marcelin Jobard, pionnier de la propriété intellectuelle qui développe les collections de manière remarquable. Son objectif est prioritairement tourné vers l'industrie et les industriels, à qui s'adresse l'établissement, et c'est dans cette perspective qu'il conçoit le musée : "Quant 
aux résultats, je n'hésite pas à croire qu'ils seraient immenses pour la Belgique ; car c'est la difficulté de se procurer des notions sur les inventions, même les plus vulgaires, qui tient l'industrie d'un pays en arrière de celle de ses voisins » (Jobard, 1840, p. 3). Quelques années après son décès, le musée perd néanmoins de son influence, pour être définitivement fermé au cours des années 1880. De nombreux musées de l'industrie sont créés de la même manière en Europe, notamment en France, à Nantes (1834 ou 36), à Epinal (1836), à Calais (autour de la dentelle, en 1841), à Saint-Etienne (1853), etc. (Cartier, 2006).

Si l'ouverture du Conservatoire national des Arts et Métiers constitue une étape majeure reliant le musée et le monde économique, celle du musée des Manufactures, intitulé plus tard South Kensington Museum, puis Victoria \& Albert Museum, à Londres, constitue un tournant de cette évolution. Le principe du musée d'art industriel est plus directement à chercher dans les suites de la première Exposition universelle de Londres de 1851, qui confirme le rôle moteur de l'économie britannique, mais aussi l'avance prise par les industriels français au niveau de la qualité des objets manufacturés. Les Britanniques, à commencer par l'un des principaux organisateurs de l'Exposition, Henry Cole, décident de concrétiser les efforts poursuivis depuis plusieurs années en vue d'améliorer leurs industries, et lancent avec le bénéfice de la World's fair le projet d'un vaste complexe comprenant une collection d'objets de références (constituée par le biais d'acquisitions d'objets, notamment ceux présentés durant l'Exposition universelle), à destination des publics ouvriers britanniques, afin de leur offrir des modèles pour améliorer la qualité de la production. La collection du South Kensington Museum, installée dans un quartier industriel, apparaît d'emblée comme une source d'inspiration majeure pour les ouvriers et les artisans. Elle constitue le prototype d'un très grand nombre de musées d'art industriels qui vont être créés à travers l'Europe (de Bruxelles à Vienne) afin d'améliorer la production manufacturière (Mairesse, 2010, pp. 49-51). La vocation première de ces établissements, que l'on rassemble de nos jours dans la catégorie des musées d'arts décoratifs, est donc d'abord économique et souvent liée au 
travail des chambres de commerce (comme à Lyon), cherchant à vitaliser l'industrie régionale ou nationale. Dès cette époque, quelques critiques voient le jour, notamment celle de l'économiste William Stanley Jevons (1883), constatant l'obsolescence rapide des productions acquises par les musées - puisque ceux-ci sont censés montrer les technologies les plus récentes - et préconisant plutôt la visite d'usines que la constitution de musées industriels par l'État. En effet, un certain nombre d'entreprises ont déjà à ce moment constitué des collections de modèles, et ouvrent parfois leurs portes au public.

La manufacture de céramique de Sèvres conçoit ainsi, dès 1800 , le principe d'une collection qui ne prendra la forme d'un musée réellement constitué qu'à partir de la décennie suivante. Les collections rassemblées "demandaient, en raison de leur nombre et de l'intérêt qu'elles présentaient déjà, à être offertes à l'étude des fabricants, des savants et des archéologues, dans un ordre qui satisfit les différentes recherches des personnes qui désiraient connaître leur histoire » (Brongniart \& Riocreux, 1845, t. III). Le public prioritaire, comme le signalent les auteurs, est bien composé par les fabricants, et ce musée, adjoint à une manufacture nationale, est considéré à l'époque comme « un petit musée modèle » (Semper, 1851), source d'inspiration pour les musées d'art industriels et notamment le Victoria \& Albert Museum. Si les développements de ce dernier musée sont assez bien connus, il est plus difficile de connaître avec précision l'historique et le développement des premiers musées d'entreprises, mais on peut estimer que ceux-ci apparaissent (et sans doute disparaissent, en fonction de la santé des firmes) à cette époque pour trois types de raisons : constituer, au même titre que Sèvres ou d'autres manufactures, une collection illustrant le patrimoine aussi bien que le savoir-faire de l'entreprise, développer des espaces d'accueil pour des clients intéressés par les productions de la maison, mais aussi, plus prosaïquement réutiliser le matériel de présentation conçu pour une exposition nationale ou universelle. Il ne faut pas l'oublier, en effet, le principe de l'exposition universelle, surtout au XIX ${ }^{\mathrm{e}}$ siècle, est d'abord lié à l'exposition de marchandises, et les constructeurs et marchands consacrent des 
moyens considérables pour y présenter au mieux leurs produits, afin de retenir les faveurs des jurys - qui consacrent ces efforts par des médailles et diplômes - et le grand public.

Le principe de la collection - du stock - est inhérent à l'entreprise de biens manufacturés, et la plupart d'entre elles conservent des spécimens deleurproduction, soitpourdesquestions de réutilisation - notamment dans le cas de la céramique ou des tissus, un même modèle pouvant perdurer durant des décennies soit pour préserver la mémoire de l'entreprise. Certains de ces documents d'archives peuvent faire l'objet d'une sobre mise en scène au sein de l'entreprise, destinée aux générations d'ouvriers qui se succèdent. Le principe de l'exposition, quant à lui, est tout aussi étroitement associé au monde de l'industrie et constitue une méthode particulière de communication par la voie d'exposition des produits de l'industrie, encore importante de nos jours, qu'il s'agisse des premiers espaces de vente dans les marchés, des passages et des boutiques, ou des grands magasins (Morgan, 2011). Les principes d'installation, d'explication, d'esthétique de la marchandise, déjà évoqués par Benjamin (1989) dans son Livre des passages, ont donné lieu à des propositions de plus en plus sophistiquées de présentation des objets, tant pour le commerce direct (dans les boutiques ou les grands magasins) que de manière indirecte, lors des expositions nationales ou universelles. Il s'agit alors d'attirer le flâneur, de mettre en valeur les produits, mais aussi, dans le cas des expositions universelles, de mettre en scène l'entreprise, de présenter son savoir-faire au reste du monde. Bref, d'un enjeu nécessitant réflexion et financement des travaux. Mais une fois la performance - par nature éphémère - réalisée, que faire de l'investissement? Il semble souvent difficile de se séparer de mobiliers et d'installations parfois remarquables, souvent onéreux et ne pouvant être revendus. Il est fort probable que nombre d'industriels soient donc repartis avec leur mobilier d'exposition afin de l'utiliser à d'autres occasions tout en l'entreposant au sein de leur entreprise. C'est ainsi que la compagnie Baltimore and Ohio Railroad, présente à l'exposition centennale de Philadelphie de 1876 et dont le pavillon, lors de la Columbian Exhibition de 1893, constitue l'un des clous de l'exposition, conserve une partie 
de son dispositif d'exposition. Un musée au sein de l'entreprise se constitue ainsi progressivement, qui est ouvert au public en 1935 et devient l'un des premiers musées américains du genre (Coleman, 1943, p. 14). De manière différente, le bâtiment et les collections réalisés par les Charbonnages de Mariemont et de Bascoup, réalisés pour l'Exposition universelle de Paris en 1889, sont offerts par leur propriétaire, Raoul Warocqué, afin de constituer un musée industriel (Mairesse, 2007). Il est assez difficile, cependant, d'établir l'histoire de ces musées « internes », qui n'ont pour la plupart pas bénéficié de publicité en dehors des entreprises et dont la mémoire s'est souvent perdue au fil des générations. Le lien entre musées et entreprise apparaît donc au niveau de la production, par les collections d'objets propres à inspirer les fabricants ; il se développe aussi par le biais de musées d'un autre type et qui ont pour la plupart disparu de nos jours : les musées commerciaux.

\section{Genèse et déclin des musées commerciaux}

Si un certain nombre de musées cherchent dès la fin du $\mathrm{XVIII}^{\mathrm{e}}$ siècle à perfectionner la qualité de l'industrie et de ses productions, d'autres s'appliquent à en améliorer la diffusion et à explorer de nouveaux marchés. Le XIX ${ }^{\mathrm{e}}$ siècle voit non seulement l'essor de la société industrielle, mais aussi celui de la distribution de sa production à travers le monde. Il n'existe cependant pas de système d'information aussi sophistiqué que ceux qui vont progressivement voir le jour tout au long du $\mathrm{XX}^{\mathrm{e}}$ siècle (téléphonie et informatique), et le principe d'une collection de données constituée par des échantillons de matières premières pouvant être acquis dans chaque pays et des objets pouvant s'y vendre, aussi bien que des moyens nécessaires à en assurer le transport, apparaît comme une solution intéressante à mettre en œuvre. Ce sera le propre des musées économiques et commerciaux. Jusqu'à la Première Guerre mondiale au moins, la logique économique du musée apparaît comme un développement logique du champ muséal. " De même qu'il s'est créé des instituts scientifiques consacrés à l'avancement de la connaissance de la Nature, il 
s'est fondé des Instituts économiques destinés à l'avancement de l'art de l'exploiter. Ce sont les Musées économiques » (Gilson, 1914, p. 124). Georges Gilson, Directeur du Musée d'Histoire naturelle de Belgique, décrit alors le principe de fonctionnement d'une catégorie de musées ayant à cette époque largement pignon sur rue : les musées économiques ou commerciaux. Leur origine remonte au début du siècle précédent avec ce qui peut être considéré comme la première école de commerce au monde. L'École supérieure de Commerce de Paris, ouverte en 1820 et transformée en 1822 sous le nom d'École spéciale de Commerce et d'Industrie, puis d'École spéciale de Commerce, est dirigée à l'époque par Brodart. C'est ce dernier, avec l'aide de Vital- Roux, qui crée un musée de marchandises ou musée commercial interne à l'école, constitué de collections d'échantillons, afin de mettre les futurs responsables commerciaux en situation concrète de négoce, dans le cadre d'approches pédagogiques nouvelles (Renouard, 1898, p. 14). On retrouve la même logique en Belgique, quelques années plus tard, à Melle-lez-Gand, avec la création d'un musée commercial au sein d'une institution scolaire tenue par les frères de la Doctrine chrétienne (Deleuil, 1887), mais ce n'est qu'au cours des années 1880 que le terme de musée commercial prend son essor, avec la création du plus célèbre d'entre eux, à Bruxelles.

C'est à partir d'une exposition nationale temporaire réalisée pour le cinquantième anniversaire de la Belgique qu'est conçu le projet d'une collection rassemblant des données propres à favoriser les exportations belges. En 1882 s'ouvre ainsi, sous les auspices du ministère des Affaires étrangères, un musée commercial réunissant, sur trois étages, des collections destinées aux importateurs et aux exportateurs (mais le musée est ouvert à tous les publics) afin de " renseigner les manufacturiers et les négociants belges sur la marche des affaires dans les pays étrangers, et de leur faciliter en même temps les transactions commerciales avec les consommateurs et les producteurs de ces mêmes contrées. Son but est également de fournir les moyens d'étudier pratiquement les affaires ; il arme le producteur pour la concurrence et cela non seulement en lui mettant sous les yeux le produit préféré dans telle ou telle partie du globe, mais 
en lui faisant connaître également dans quelles conditions ce produit peut trouver à s'écouler [...] » (Des Tournelles, 1888, pp. 9-10). Le musée, équipé d'un bureau de renseignements et d'une bibliothèque, publie également un bulletin hebdomadaire. On prétend que le Musée commercial de Bruxelles aurait rapporté (de manière indirecte, car sa fréquentation est gratuite) des profits plus importants que ceux générés par la modernisation du port d'Anvers. Progressivement, de nombreux pays s'équipent d'institutions similaires, aux Pays-Bas, en Allemagne, en Angleterre, en Hongrie ou au Portugal et en Suisse (Des Tournelles, 1888). En France, quelques initiatives privées ou patronnées par la chambre de commerce voient le jour, notamment à Lille, à Amiens ou à Bordeaux. L'un des établissements les plus importants de ce type est inauguré à Philadelphie au tournant du $\mathrm{XX}^{\mathrm{e}}$ siècle, présentant la même logique à travers des collections s'étendant sur plusieurs milliers de $\mathrm{m}^{2}$ - il ne fermera ses portes qu'en 1994 (Conn, 2010 pp. 172-196). La plupart de ces musées, en revanche, disparaissent après la Première Guerre mondiale, remplacés par d'autres dispositifs plus modernes d'aide au commerce extérieur - les collections d'échantillons, si elles ont le mérite de concrétiser l'approche livresque s'avèrent rapidement obsolètes, comme le suggérait déjà Jevons (1883).

Le paysage muséal qui se présente après la Première Guerre mondiale apparait ainsi assez différent de celui qu'il offrait tout au long du XIX ${ }^{\mathrm{e}}$ siècle. Presque tous les musées industriels ou d'art industriel ont vu leur qualification révisée en musées d'art décoratif, en cela plus proche d'une certaine logique muséale fondée sur le beau et la connaissance, et moins sur ses liens avec l'économie. La plupart des musées commerciaux, en revanche, disparaissent avec leurs collections. C'est dans ce contexte que se développent les musées d'entreprise, phénomène alors suffisamment important pour que Laurence Vail Coleman, Directeur de l'association américaine des musées, leur consacre un ouvrage les répertoriant (Coleman, 1943). L'auteur souligne alors les multiples objectifs de ce type de musées, qui servent aussi bien de manière interne, pour stimuler la fierté des travailleurs, mais aussi pour préserver la mémoire des productions (et le cas échéant 
attester leur authenticité) et du développement de la société, que, de manière externe, améliorer l'image de l'entreprise en recevant les meilleurs clients - et parfois le grand public - dans un contexte de relations publiques et de communication d'entreprise. C'est probablement à ce moment que l'on peut commencer à parler des musées d'entreprise comme d'une catégorie spécifique, du moins aux États-Unis, car dans la plupart des pays européens, ce mouvement de reconnaissance semble prendre plus de temps, au point de se demander s'il est même réellement effectif de nos jours.

\section{Conclusions}

Catégorie contestée car liée au monde des entreprises et à la logique de marché, les musées d'entreprise apparaissent comme une création relativement récente, voire une catégorie sans réelle histoire, la fortune de ces musées évoluant au gré du succès commercial ou de la faillite des entreprises qui les abritent. L'histoire des musées est cependant nettement plus complexe que celle qu'illustrent des établissements comme le Louvre ou les muséums d'histoire naturelle; elle montre aussi la relation étroite qu'entretiennent depuis leur origine les musées avec l'économie. Les propos de Leibniz soulignent d'emblée la nécessité de réfléchir sur les fins du musée, et notamment sa logique économique. Pour prospérer, ces établissements doivent montrer leur utilité (Dana, 1920), et si celle-ci est surtout présentée comme indirecte, participant à la formation des habitants d'un pays ou d'une région, voire à la qualité de vie d'un lieu ou comme symbole de sa prospérité, elle peut également être présentée comme directe, sur le plan économique. Ce dernier principe a particulièrement été mis en valeur ces dernières années, et le monde des musées est de plus en plus souvent présenté, à l'image du Musée Guggenheim de Bilbao, comme une infrastructure dont les répercussions sur l'économie locale, via le tourisme et le développement de la créativité, sont considérables (KEA, 2006).

Ce lien entre économie et patrimoine est plus ancien : la collection muséale est d'emblée présentée, lors de la 
constitution du Conservatoire des Arts et Métiers, comme une base de données de modèles et de technologies constituée par les générations précédentes, dont la consultation permettra d'améliorer les performances de l'industrie. On retrouve les mêmes principes économiques dans les expositions nationales de l'industrie, puis avec la première Exposition universelle en 1851 (Schroeder- Gudehus, 1992). C'est un principe similaire qui sera développé, par les musées d'art industriel, qui se disséminent à travers l'Occident tout au long du XIX ${ }^{\mathrm{e}}$ siècle. Le lien peut-être le plus pur que l'on peut trouver entre musées et économie est sans doute obtenu avec les musées commerciaux, dont le principe émerge avec la création de la première école de commerce en France et qui connaissent leur apogée peu de temps avant la Première Guerre mondiale. D'emblée, les musées d'entreprises apparaissent - à l'instar des musées que l'on retrouve dans les grandes manufactures publiques, par exemple à Sèvres - comme des établissements participant, d'une manière ou d'une autre, au développement économique de leur pays. Si les pouvoirs publics ont décidé de créer un certain nombre d'institutions à portée nationale, afin de développer l'industrie et les manufactures, c'est en étant accompagnés par de multiples initiatives privées, soit par le biais des chambres de commerce, soit par des entreprises spécifiques.

L'intervention massive des pouvoirs publics, durant les trente glorieuses, à l'aune de l'hégémonie des politiques keynésiennes, a largement favorisé, entre la Seconde Guerre mondiale et la fin des années 1980, une vision des musées fondée sur un soutien essentiellement public et éloignée des enjeux et des contraintes économiques. Le tournant commercial des musées, à la fin des années 1980, dans le sillage du développement de politiques néolibérales (Harvey, 2005), a partiellement rebattu les cartes, réintroduisant en partie la logique de marché au sein du monde muséal. Les liens du musée avec l'économie, désormais, apparaissent avec une certaine évidence afin d'assurer la pérennité de l'institution.

Si le musée d'entreprise a partiellement obtenu une meilleure reconnaissance, en regard des décennies précédentes, il reste à 
l'écart par rapport aux musées " classiques » constituant le cœur de l'institution muséale. Son histoire apparaît ainsi le plus souvent comme négligeable en regard de celle des musées classiques et essentiellement liée aux projets commerciaux d'une entreprise ou à la volonté d'un patron collectionneur. Les éléments présentés dans cet article permettent de rattacher le projet du musée d'entreprise à une logique plus ancienne, adoptée à des niveaux divers, à son origine, par nombre de musées techniques et industriels, par les musées commerciaux ainsi que par les musées d'art décoratif et industriel. Ces derniers établissements ont à leur tour évolué ou ont progressivement pris des chemins divergents. Il n'en reste pas moins qu'ils partagent avec ces musées d'entreprise une origine partiellement commune.

\section{Références}

Benjamin, W. (1989). Paris, capitale du XIXe siècle. Le livre des passages, Paris : Cerf. Bredekamp, H. (1997), Machines et cabinets de curiosité, Paris : Diderot éditeur.

Brongniart, A. \& Riocreux, D. (1845). Description méthodique du musée céramique de Sèvres, Paris : Leleux.

Coleman, L.V. (1943). Company Museums, Washington: American Association of Museums.

Colmon, A. de (1855). Histoire des expositions des produits de l'industrie française, Paris : Guillaumin.

Conn, S. (2010). Do Museums still Need Objects?, Philadelphia : University of Pennsylvania Press.

Cartier, C. (2006). « Des musées pour l'industrie », in Daumas J.-C., La mémoire de l'industrie, Besançon : Presses universitaires de Franche-Comté, 162-183.

Dana; J.C. (1920). A Plan for a New Museum - The Kind of Museum it Will Profit a City to Maintain, Woodstock: Elm Tree Press.

Deleuil, A. (1887). Annuaire des musées commerciaux français à l'étranger, Avignon : Imprimerie administrative.

Des Tournelles, F. (1888). Les musées commerciaux à l'étranger. Rapport de mission présenté à M. le Sous-secrétaire d'État aux colonies. Paris : Ministère de la Marine et des Colonies, Chalamel et Cie éditeurs.

Foucher de Careil, A. (1875). Oeuvres de Leibniz, Paris : Firmin Didot.

Gilson, G. (1914). Le musée d'histoire naturelle moderne - Sa mission, son organisation, ses droits, Bruxelles : Hayez.

Gregoire, H. (1794). Rapport sur l'établissement d'un conservatoire des arts et métiers, Paris : Imprimerie nationale, Vendémiaire An III.

Harvey, D. (2005). A brief History of Neoliberalism, Oxford: Oxford University Press. 
ICOM (2016). Statuts de l'ICOM, Paris, Disponible sur Internet: http://icom.museum/ fileadmin/user_upload/pdf/Statuts/2016_Statutes_FR.pdf

Jacomy, B. (1995). « Du cabinet au Conservatoire. Les instruments scientifiques du Conservatoire des Arts et Métiers à Paris », Journal of the History of Collections, 7, 227-233.

Jevons, W.S. (1883). "The use and abuse of museums », Methods of social reforms and other papers, London: MacMillan, 52-81.

Jobard, J.-B.-A.-M. (1840). Plan d'organisation du Musée de l'industrie, présenté au ministre de l'intérieur, Bruxelles.

KEA (2006). Study on the economy of culture in Europe, Bruxelles, Etude conduite pour la Commission européenne. Disponible sur Internet: http://ec.europa.eu/assets/ eac/culture/library/studies/cultural-economy_en.pdf

Labourdette, M.-C. (2015). Les musées de France, Paris, Presses universitaires de France (Que sais-je ?).

Mairesse, F. (2002), Le musée, temple spectaculaire, Lyon, Presses universitaires de Lyon.

Mairesse, F. (2010). Le musée hybride, Paris : La Documentation française.

Mairesse, F. (2007). Mariemont, capitale du don : des Warocqué aux Amis de Mariemont, Morlanwelz, Musée royal de Mariemont.

Michel, H. (1967). « Canzius Onderwijngaart et le Musée de l'Industrie à Bruxelles, 1827-1887 », Janus, LIV,182-188.

Morgan, T. (2011). Visual Merchandising. Window and in-store displays for retailing, London: Laurence King.

Poulot, D. (1997). Musée, nation, patrimoine, Paris : Gallimard.

Pousseur, J.M. (1988). Bacon, inventer la science, Paris : Belin.

Rasse, P. (2017). Le musée réinventé, culture patrimoine, médiation, Paris : CNRS Édition.

Renouard, A. (1898). Histoire de l'école supérieure de commerce de Paris (18201898), Paris : Au siège de l'association.

Schroeder-Gudehus, B. (Dir.) (1992). La société industrielle et ses musées : demande sociale et choix politiques 1890-1990, Paris : Éd. des archives contemporaines.

Semper, G. (1851), Science, industrie et art, Paris : Infolio [2012].

Smith, A. (1776). Recherches sur la nature et les causes de la richesse des nations, Paris : Flammarion [1991], 2 vol.

Société d'encouragement (1810). "Séance générale u 8 août 1810 », Bulletin de la société d'encouragement pour l'industrie nationale, août, LXXIV.

Société d'ingénieurs et. al. (publié par) (1849). Musée industriel, agricole et artistique, ou description de l'exposition faite à Paris en 1849, Paris : au bureau de l'administration.

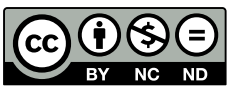

Publié sous la licence Creative Commons

«Attribution - Pas d'Utilisation Commerciale - Pas de Modification 4.0 International» (CC BY-NC-ND) 